\title{
DESEMPENHO DE GRADUANDOS EM MEDICINA VETERINÁRIA DA USP NA DISCIPLINA DE INSPEÇÃO SANITÁRIA DOS PRODUTOS DE ORIGEM ANIMAL, A PARTIR DA MODERNIZAÇÃO DE METODOLOGIAS DE ENSINO E AVALIAÇÃO, ENTRE OS ANOS DE 2017 E 2020
}

\author{
Victor Chiaroni Galvão ${ }^{1}$ \\ Mestre em Ciências pelo Departamento de Medicina Veterinária Preventiva e Saúde Animal \\ da FMVZ-USP e Docente da Disciplina de Higiene e Inspeção Sanitária dos Produtos de \\ Origem Animal da Universidade Paulista - Campus Anchieta, São Paulo, Brasil \\ https://orcid.org/0000-0003-1293-7119 \\ vchiaroni@gmail.com
}

\section{Simone de Carvalho Balian ${ }^{2}$}

Prof ${ }^{a}$.Dra . Disciplina de Inspeção Sanitária dos Produtos de Origem Animal da Faculdade de Medicina Veterinária e Zootecnia da Universidade de São Paulo, São Paulo, Brasil https://orcid.org/0000-0002-2211-9315 balian@usp.br

\footnotetext{
${ }^{1}$ Participou ativamente da redação e elaboração do artigo, discussão dos resultados e revisou e aprovação da versão final do trabalho.

${ }_{2}^{2}$ Participou ativamente da discussão dos resultados e revisou e aprovação da versão final do trabalho.
}

\section{RESUMO}

Este relato de caso teve como objetivo avaliar o desempenho de graduandos em Medicina Veterinária da Universidade de São Paulo na disciplina de Inspeção Sanitária dos Produtos de Origem Animal quando da aplicação de novas metodologias de estruturação, desenvolvimento e avaliação de conteúdos organizados por assuntos, em blocos. Ao final de cada bloco foi feita uma prova teste de múltipla escolha. A amostra deste estudo foi formada por 335 alunos do 9 응 semestre de medicina veterinária entre os anos 2017, 2018, 2019 e 2020. Foram utilizadas análise estatística descritiva e multivariada (teste $t$ ) dos dados. Constatou-se que houve aumento das notas finais entre os anos de 2018, 2019 e 2020 em relação ao ano de 2017, quando ainda não se utilizava esta abordagem. O resultado do teste $t$ foi de $t$ $=1,12^{-24}(2017-2018), t=2,06^{-15}$ (20172019) e $t=6,55^{-15}(2017-2020)$, com $p<0,05$, evidenciando diferença estatística entre as médias das notas dos alunos de 2017, 2018, 2019 e 2020. Concluiu-se que a organização dos conteúdos em blocos e a aplicação de avaliação permitiu a melhoria de desempenho dos estudantes na disciplina.

Palavra-chave: Aprendizagem. Avaliação somativa. Teste de múltipla escolha.

\footnotetext{
ABSTRACT

This case report aimed to evaluate the performance of undergraduate students in Veterinary Medicine at the University of São Paulo in the discipline of Sanitary Inspection of Products of Animal Origin when applying new methodologies for structuring, developing and evaluating content organized by block subjects. At the end of each block, a multiple-choice test was performed. The sample of this study was formed by 335 students from the 9th semester of veterinary medicine between the years 2017, 2018, 2019 and 2020.
} 
Descriptive and multivariate statistical analysis ( $t$ test) of the data was used. It was found that there was an increase in the final grades between the years 2018, 2019 and 2020 compared to the year 2017 , when this approach was not yet used. The result of the $t$ test was $t=1,12^{-24}(2017-2018), t=$ $2,06^{-15}(2017-2019)$ and $t=6,55^{-15}$ (2017$2020)$, with $p<0,05$, showing a statistical difference between the average grades of students in 2017, 2018, 2019 and 2020. It was concluded that the organization of the contents in blocks and the evaluation application allowed the improvement of students' performance in the discipline.

Keyword: Learning. Summative Assessment. Multiple choice test.

\section{INTRODUÇÃO}

A Organização Mundial de Saúde Animal, através do seu documento sobre diretrizes curriculares para a graduação em Medicina Veterinária (OIE, 2013), define que Segurança dos Alimentos é garantir a inocuidade dos alimentos do estábulo à mesa. A Faculdade de Medicina Veterinária e Zootecnia da Universidade de São Paulo (FMVZUSP) em sua disciplina de Inspeção Sanitária dos Produtos de origem Animal (ISPOA) segue as diretrizes da OIE. Outras universidades pelo mundo também adotaram o mesmo princípio (KYRIAKIS, 2015). O, discente da FMVZ-USP, portanto, é formado para ter uma visão ampla sobre a cadeia de produção dos alimentos a fim de intervir com soluções adequadas à Saúde Pública e à viabilidade econômica.

A disciplina de ISPOA faz parte do conjunto de conhecimentos referentes à Segurança dos Alimentos (LUDÉN, 2007; SMULDERS, 2012) e é uma disciplina obrigatória nos cursos de Medicina Veterinária no Brasil (BRASIL, 2019). Tem por propósito instrumentalizar o graduando para promover a Saúde Pública por meio do estudo sistemático da cadeia de alimentos sobre a ótica da inocuidade e viabilidade econômica. Os veterinários que atuarão no mercado de trabalho relacionado à ISPOA irão inspecionar desde os animais vivos que foram encaminhados ao abate até produtos como mel, ovos, leite e seus derivados, pescado e seus derivados também. É conteúdo obrigatório ao profissional da ISPOA conhecer os programas de autocontrole, tais como Boas Práticas de Fabricação (BPF) e Análise de Perigos e Pontos Críticos de Controle (APPCC), além de microbiologia e tecnologia de alimentos. Também devem desenvolver habilidades humanas para a liderança de pessoas e gestão de processos (YIANNAS, 2009; GRIFFITH, LIVESEY e CLAYTON, 2010), envolvendo questões relacionadas à comunicação 
e à avaliação de comportamentos de risco para a contaminação dos alimentos.

Para estimular graduandos em Medicina Veterinária na FMVZ-USP e mantê-los conectados às aulas e seus conteúdos ao longo do semestre na disciplina ISPOA, a partir de 2018 adotaram-se novas metodologias de ensino e avaliação de desempenho dos mesmos com relação à prática anterior (até 2017) quando se apresentava as aulas, distribuídas ao longo do semestre e fazia-se uma prova com $50 \%$ do conteúdo e outra com $100 \%$ do conteúdo, obtendo como conceito final da disciplina, a média simples das duas notas.

\section{MÉTODO}

A amostra referente à nova abordagem de ensino e avaliação, totalizou 335 graduandos do 9응 semestre em Medicina Veterinária da USP, compreendendo 88 em 2018; 76 em 2019 e 91 em 2020. A amostra relativa à metodologia tradicional, em 2017 conteve 80 estudantes.

Em 2017 a disciplina continha 90 horas de carga horária distribuídas em aulas teóricas, práticas e visitas técnicas. O método de avaliação da disciplina consistia em um trabalho em grupo e uma avaliação final de todo o conteúdo. A disciplina teve a participação de pós-graduando do Programa de Aperfeiçoamento de Ensino da USP (PAE) a partir de 2016 para o auxílio nas atividades relativas à disciplina.

$$
\text { Em 2018, } 2019 \text { e } 2020 \text { a }
$$

disciplina modernizou a metodologia de ensino, criando blocos de conteúdos e aplicando avaliações específicas para cada bloco, gerando várias notas ao longo do semestre. Essa prática organizou os temas, permitiu aos estudantes perceber 0 avanço da disciplina, iniciando e finalizando os blocos, permitindo-lhes várias oportunidades para avaliação e obtenção de pontos ao longo do semestre. OS conteúdos correlatos foram agrupados e os assuntos que não se relacionavam diretamente entre si também foram agrupados. Por exemplo, a inspeção ante e post mortem, técnicas de insensibilização e abate de animais de açougue formaram um bloco de conteúdo. Entretanto, inspeção de produtos de abelha, pescado e produtos não comestíveis formaram outro bloco, assim sucessivamente, formando-se quatro 
blocos de conteúdos e quatro avaliações.

A avaliação de aprendizagem ocorreu ao final de cada bloco, por meio da Prova Teste de Múltipla Escolha (PTME) constituída de quatro a seis questões. $\mathrm{O}$ conjunto de questões pertencia a concursos públicos direcionados a Médicos Veterinários e questões elaboradas pelo docente responsável pela disciplina. As questões foram selecionadas de acordo com o conteúdo do bloco. O objetivo da aplicação da avaliação em blocos de conteúdo foi estimular os alunos a testarem os seus conhecimentos sobre o bloco abordado até então.

Durante a aplicação da PTME ao fim de cada bloco, os alunos foram dispostos em fileiras intercaladas para realizar a PTME sem consulta (I). Ao término da prova o aluno entregou ao pós-graduando PAE o gabarito das questões e permanecia com a folha de questões (II). Os alunos foram instruídos a fazerem grupos (8-10 pessoas) para discutirem as respostas (III). Terminando a discussão, as questões e as respectivas respostas foram apresentadas em projeção de slides para discussão com o docente e o aluno PAE (IV). O tempo de cada etapa do método foi, aproximadamente: (I) 15-20 min, (II) e (III) 5 min e (IV) 15-30 min.

As análises estatísticas descritiva e multivariada (teste $t$ ) foram aplicadas nos dados para extrair inferências. Foi utilizado Microsoft $E_{x c e}{ }^{\circledR} 2010$ para compilação dos dados e análise estatística.

O presente estudo é a continuidade do trabalho publicado anteriormente por Galvão e Balian (2020) que encontraram evidências positivas da aplicação do referido método e da reformulação da disciplina.

\section{RESULTADOS E DISCUSSÃO}

Após a tabulação e análise dos dados, obtiveram-se os resultados contidos na Tabela 1. Houve aumento das notas finais entre os anos de 2017 e 2020, após aplicação do método PTME. A menor nota foi de 3,6 pontos no ano de 2017, inferior à média mínima para aprovação na disciplina $(5,0)$, porém, em 2020 também houve nota abaixo da média (4,3 pontos). Já as maiores notas pertencem aos anos 2018, 2019 e 2020. Entre os anos de 2018 a 2020, a média geral das turmas aumentou pelo menos 1,3 pontos em 
relação à média de 2017 , o que é significativo.

Tabela 1 - Média alcançada pelos alunos e notas mais altas e mais baixas obtidas entre os anos 2017 e 2020.

\begin{tabular}{ccccc}
\hline Ano & $\begin{array}{c}\text { Menor } \\
\text { nota }\end{array}$ & $\begin{array}{c}\text { Maior } \\
\text { nota }\end{array}$ & $\begin{array}{c}\text { Média } \\
\text { da } \\
\text { turma }\end{array}$ & $\begin{array}{c}\text { Desvio } \\
\text { Padrão }\end{array}$ \\
\hline 2017 & 3,6 & 9,3 & 7,3 & 1,0 \\
2018 & 7,4 & 10,0 & 9,0 & 0,5 \\
2019 & 7,1 & 9,8 & 8,6 & 0,6 \\
2020 & 4,3 & 10,0 & 9,0 & 1,1 \\
\hline
\end{tabular}

A Tabela 2 apresenta intervalos de nota final dos alunos entre os anos de 2017 e 2020. Nota-se que no ano de 2017 houve quatro alunos que obtiveram notas inferiores a 5,0 pontos (reprovação). Já no ano de 2018 e 2019 não houve notas inferiores a 5,0 pontos, enquanto em 2020 obteve-se apenas uma. Em 2017, 20 alunos apresentaram notas entre 5,0 e 7,0, já nos anos de 2018 e 2019 nenhum aluno teve notas nesse intervalo, porém em 2020 seis alunos encontraram-se nessa condição. No intervalo entre 7,1 e 9,0, o ano de 2017 apresentou 55 alunos, enquanto em 2018 verificaramse 42 alunos; em 2019 sessenta alunos e em 202041 alunos nesse intervalo. Quando se observa notas maiores que 9,0 pontos, entretanto, o ano de 2018 se destacou, apresentando 46 alunos, seguido pelo ano de 2020 com 43 e
2019 com 16. Já no ano de 2017 apenas um aluno obteve nota superior a 9,0 pontos.

Tabela 2 - Distribuição das notas dos alunos da turma entre os anos 2017 e 2020.

\begin{tabular}{|c|c|c|}
\hline Ano & $\begin{array}{c}\text { Intervalo } \\
\text { de nota }\end{array}$ & $\begin{array}{l}\text { Número } \\
\text { de alunos }\end{array}$ \\
\hline \multirow[t]{4}{*}{2017} & $<5,0$ & 4 \\
\hline & $5,0-7,0$ & 20 \\
\hline & $7,1-9,0$ & 55 \\
\hline & $>9,0$ & 1 \\
\hline Total & & 80 \\
\hline \multirow[t]{4}{*}{2018} & $<5,0$ & 0 \\
\hline & $5,0-7,0$ & 0 \\
\hline & $7,1-9,0$ & 42 \\
\hline & $>9,0$ & 46 \\
\hline Total & & 88 \\
\hline \multirow[t]{4}{*}{2019} & $<5,0$ & 0 \\
\hline & $5,0-7,0$ & 0 \\
\hline & $7,1-9,0$ & 60 \\
\hline & $>9,0$ & 16 \\
\hline Total & & 76 \\
\hline \multirow[t]{4}{*}{2020} & $<5,0$ & 1 \\
\hline & $5,0-7,0$ & 6 \\
\hline & $7,1-9,0$ & 41 \\
\hline & $>9,0$ & 43 \\
\hline Total & & 91 \\
\hline
\end{tabular}

Os dados apresentados indicam que 0 desempenho dos alunos aumentou de um ano para o outro. Acredita-se que a fragmentação de forma organizada dos conteúdos e das avaliações tenham proporcionado aos 
estudantes maior ordenação e aproveitamento durante a disciplina.

A fim de verificar se houve diferença estatística entre as médias de notas finais das turmas de 2017 e dos outros anos após aplicação do método PTME foi aplicado o teste $t$ de Student, com intervalo de confiança de 95\%, entendendo que a hipótese nula $(\mathrm{HO})$ indicaria que não houve diferença estatística entre as médias de notas das turmas de 2017 e os outros anos, e a hipótese alternativa $(\mathrm{H} 1)$ que existe diferença entre as médias dos alunos (HAIR, 2009).

A Tabela 3 apresenta os resultados do teste $t$ entre os anos 2017 e 2020. Houve diferença estatística, $p<0,05$ levando ao aceite da hipótese $\mathrm{H} 1$, isto é, houve diferença entre as médias das notas finais dos alunos de 2017 e dos anos posteriores. Este fato indica que a modernização da metodologia de ensino e avaliação adotadas na disciplina de ISPOA contribuiu para a melhoria de performance dos graduandos, no que se refere as suas notas.

Tabela 3 - Teste $t$ Student sobre as notas entre os anos 2017 e 2020.

\begin{tabular}{ccc}
\hline Anos & p-valor & $\begin{array}{c}\text { Estatística } \\
\text { do teste } \boldsymbol{t}\end{array}$ \\
\hline $2017-2018$ & $1,12^{-24}$ & 12,14
\end{tabular}

$2017-2019 \quad 2,06^{-15} \quad 8,84$

$2017-2020 \quad 6,55^{-15} \quad 8,56$

A fim de aprofundar o estudo do desempenho dos alunos, a Tabela 4 apresenta os dados de menor nota, maior nota, média, mediana e desvio padrão entre os anos 2018 e 2020, apenas sobre as notas da PTME. Notase que os resultados de menor desempenho ocorreu nos anos 2018 e 2020, sendo 2019 o melhor resultado. Em relação à maior nota obtida na turma, em todos os anos houve nota máxima (10,0 pontos). A média da turma apresentou gradativo aumento com o passar dos anos. Por fim, o desvio padrão apresentou redução gradativa de resultado indicando pouca variação em torno da média.

Tabela 4 - Média alcançada pelos alunos e notas mais altas e mais baixas obtidas nas PTME entre os anos 2018 e 2020.

\begin{tabular}{ccccc}
\hline Ano & $\begin{array}{c}\text { Menor } \\
\text { nota }\end{array}$ & $\begin{array}{c}\text { Maior } \\
\text { nota }\end{array}$ & $\begin{array}{c}\text { Média } \\
\text { da } \\
\text { turma }\end{array}$ & $\begin{array}{c}\text { Desvio } \\
\text { Padrão }\end{array}$ \\
\hline 2018 & 4 & 10 & 8,4 & 1,2 \\
2019 & 5,3 & 10 & 8,7 & 1,0 \\
2020 & 4,5 & 10 & 9,2 & 0,9 \\
\hline
\end{tabular}

Observando a Tabela 5, que apresenta a distribuição das notas dos alunos na PTME entre os anos 2018 e 
2020, houve poucos alunos que obtiveram nota inferior a 5,0 pontos. No intervalo de notas entre 5,0 e 7,0 pontos, o ano de 2018 apresentou 11 alunos, enquanto nos anos de 2019 e 2020 apenas três em cada ano. Já na categoria de 7,1 a 9,0 pontos, nota-se que em 2018 e 2019 a maioria dos alunos pertenciam a esse intervalo de notas (cerca de 40 alunos); no ano de 2020 houve 37. Acima de 9,0 pontos, observa-se que a quantidade de alunos aumentou ano a ano, em 2018 com 28 alunos, 2019 com 31 alunos e 2020 com 49 alunos.

Tabela 5 - Distribuição das notas dos alunos na PTME da turma entre os anos 2018 e 2020.

\begin{tabular}{ccc}
\hline Ano & $\begin{array}{c}\text { Intervalo } \\
\text { de nota }\end{array}$ & $\begin{array}{c}\text { Número } \\
\text { de alunos }\end{array}$ \\
\hline 2018 & $<5,0$ & 1 \\
& $5,0-7,0$ & 11 \\
& $7,1-9,0$ & 48 \\
Total & $>9,0$ & 28 \\
& & 88 \\
2019 & $<5,0$ & 0 \\
& $5,0-7,0$ & 3 \\
& $7,1-9,0$ & 42 \\
& $>9,0$ & 31 \\
Total & & 76 \\
& & \\
& & 1 \\
& & 3 \\
& $5,0-7,0$ & 37 \\
& $7,1-9,0$ & 49 \\
\hline
\end{tabular}

Total

90

Os valores do teste $t$ de Student contidos na Tabela 6 demonstram que ocorreu diferença estatística $(p<0,05)$ entre os anos em relação às notas na PTME. A maior diferença estatística foi entre os anos 2018 e 2020, o que corrobora com os resultados obtidos anteriormente sobre a distribuição das notas e as medidas de tendência central.

Tabela 6 - Teste $t$ Student sobre as notas da PTME entre os anos 2018 e 2020.

\begin{tabular}{ccc}
\hline Anos & p-valor & $\begin{array}{c}\text { Estatística } \\
\text { do teste } \boldsymbol{t}\end{array}$ \\
\hline $2018-2019$ & 0,03 & 2,14 \\
$2018-2020$ & $2,55^{-6}$ & 4,86 \\
$2019-2020$ & 0,01 & 2,83 \\
\hline
\end{tabular}

A intenção de reorganizar a disciplina de ISPOA em blocos de conteúdo foi fruto de avaliações formativas realizadas pelo docente responsável e pelo pós-graduando PAE, buscando aprimorar o processo de ensino-aprendizagem através de ajustes e atualizações metodológicas (SILVA, 2014; REYNOLDS, 2008; BALLESTER, 2003).

Ao final de cada bloco de conteúdo foi aplicado PTME. Sabe-se 
que testes de múltipla escolha fazem parte da avaliação somativa. A avaliação somativa visa avaliar se os propósitos, progressos e resultados dos avaliados estão de acordo com os objetivos do programa ou de uma disciplina (REYNOLDS, 2008). Consiste na soma de um ou mais resultados de avaliação. Silva (2014) afirma que avaliações frequentes e consistentes melhoram o rendimento dos alunos. De fato, o incremento das PTME resultou em aumento do desempenho, constatado neste estudo.

Quanto à avaliação do tipo múltipla escolha, Bollela (2018) apresenta suas vantagens e desvantagens. Entre as vantagens destacam-se a possibilidade de avaliar os alunos em diferentes níveis de complexidade, a facilidade de correção e feedback de desempenho de cada aluno. Essas vantagens favoreceram a metodologia do presente trabalho, por isso da escolha dos testes múltipla escolha e não outra forma de avaliação. Já as desvantagens seriam a dificuldade em se avaliar a profundidade de raciocínio, a dificuldade de delimitação estreita e precisa em apenas uma questão de tal assunto da disciplina e o acerto causal ou casual (BOLLELA, 2018).
Realmente não se consegue alcançar os raciocínios em profundidade dos alunos com PTME, porém a discussão das questões imediatamente após a realização da prova (feedback) permite ampliar o conhecimento, esclarecer dúvidas e aprofundar o tema em alguns casos. A delimitação dos assuntos das questões foi facilitada pela ampla gama de questões de concursos públicos veterinários disponíveis na internet.

Importante salientar que 0 feedback permite aos participantes identificar os erros cometidos e fazer sua autoavaliação pessoal, reconhecendo sua própria performance no curso, além da pontuação obtida. Para fins de prática educacional, é útil observar que o feedback tende a ser eficaz, independentemente de ser dado aos graduandos imediatamente ou após um atraso (BUTLER, 2018).

\section{CONCLUSÕES}

A modernização da metodologia de ensino, organizando os conteúdos em blocos por afinidade de temas e a aplicação das PTME após cada um dos blocos mostrou aumento de desempenho de graduandos, quando comparados com estudantes que receberam os conteúdos de forma 
contínua e tiveram uma ou duas provas considerando todo o conteúdo da disciplina.

Acredita-se que 0 bom desempenho da metodologia obtida na disciplina de ISPOA possa ser aplicada em outras e até mesmo em outros cursos de graduação.

\section{REFERÊNCIAS}

BALLESTER, M. Avaliação como apoio à aprendizagem. Porto Alegre: Editora Artmed, 2003.

BOLLELA, V. R.; BORGES, M. de C.; TRONCON, L. E. de A. Avaliação somativa de habilidades cognitivas: experiência envolvendo boas práticas para a elaboração de testes de múltipla escolha e a composição de exames. Revista Brasileira de Educação Médica. v. 4, n. 42, 2018, p. 74-85.

BRASIL. Ministério da Educação. Conselho Nacional de Educação. Câmara de Educação Superior. Resolução $N^{\circ} 3$, de 15 de agosto de 2019. Institui as Diretrizes Curriculares Nacionais do Curso de Graduação em Medicina Veterinária e dá outras providências. Diário Oficial [da] União, Brasília, 16 de ago. 2019. Seção I, p. 199.
BUTLER, A. C. Multiple-Choice Testing in Education: Are the Best Practices for Assessment Also Good for Learning? Journal of Applied Research in Memory and Cognition. n. 7, p. 323331, 2018.

GALVÃO, V. C.; BALIAN, S. de C. Disciplina de Inspeção Sanitária: Alteração de Cronograma e Avaliação de Graduandos em Medicina Veterinária. Revista de Graduação USP. v. 4, n. 1, 2020.

GRIFFITH, C. J.; LIVESEY, K. M.; CLAYTON, D. A. Food safety culture: the evolution of an emerging risk factor? British Food Journal. v. 112, n. 4, p. 426-438. 2010. Disponível em: < https://doi.org/10.1108/000707010110 34439>. Acesso em: 20 Jan. 2021. HAIR, J.; ANDERSON, R.; BABIN, B. Multivariate Data Analysis: a global perspective. 7. ed. Upper Saddle River: Prentice Hall, 2009. 785 p.

LUNDÉN, J.; BJORKROTH, J.; KORKEALA, H. Meat Inspection Education in Finnish Veterinary Curriculum. Journal of Veterinary Medicine Educational. v. 2, n. 34, 2007.

KYRIAKIS, S. C.; ALEXOPOULOS, C.; TASSIS, P. D.; TZIKA, E. D.; KRITAS, S. K.; BURRIEL, A. R. Future Directions in the European Union for Veterinary 
Education as Related to Food-

Producing Animals, with Special

Reference to Greece. Journal of

Veterinary Medicine Educational. v. 1, n. 31, 2015.

REYNOLDS, C. R.; LIVINGSTON, R. B.; WILLSON, V. Measurement and Assessment in Education. 2. ed. Texas: Editora Pearson, 2008.

SILVA, D. S. G. da; MATOS, P. M. de S.; ALMEIDA, D. M. de. Métodos avaliativos no processo de ensino e aprendizagem: uma revisão. Cadernos de Educação | FaE/PPGE/UFPel. n. 47, p. 73-84. 2014. Disponível em: <http://dx.doi.org/10.15210/caduc.v0i4 7.4651>. Acessado em 10 ago. 2020. SMULDERS, F. J. M.; BUNCIC, S.; FEHLHABER, K.; HUEY, R. J.; KORKEALA, H.; PRIETO, M.; STEINHAUSEROVA, I. Toward Harmonization of the European Food Hygiene/Veterinary Public Health Curriculum. Journal of Veterinary Medicine Educational. v. 2, n. 39, 2012.

World Organisation for Animal Health (OIE). Veterinary Education Core Curriculum. Paris, 2013. Disponível em: $<$ http://www.oie.int/Veterinary_Educati on_Core_Curriculum.pdf $>$. Acessado em 27 mar. 2019.
YIANNAS, F. Food safety culture: creating a behavior-based food safety management system. New York: Springer-Verlag, 2009. p. 96. eBook ISBN: 978-0-387-72867-4. 